\title{
Performance enhancement of audio transmission based on LMMSE method
}

\author{
Marwa Al-Sultani ${ }^{1}$, Haraa Raheem Hatem ${ }^{2}$, Wurod Qasim Mohamed ${ }^{3}$ \\ ${ }^{1,2}$ Department of Communications Engineering, University of Diyala, Diyala, Iraq \\ ${ }^{3}$ Department of Electronic Engineering, University of Diyala, Diyala, Iraq
}

\begin{tabular}{l} 
Article Info \\
\hline Article history: \\
Received Aug 21, 202 \\
Revised Oct 3, 2020 \\
Accepted Oct 17, 2020 \\
\hline Keywords: \\
Audio transmission \\
BPSK \\
Capacity \\
Interleaving \\
Linear bloke code \\
LMMSE method \\
QPSK
\end{tabular}

Article Info

Article history:

Received Aug 21, 2020

Revised Oct 3, 2020

Accepted Oct 17, 2020

Keywords:

Audio transmission

Linear bloke code

QPSK

\begin{abstract}
The research in wireless communication has developed rapidly for the last decades as a result of raising the demand for efficient data transmission with more security and accuracy. This paper proposed a system based on the special multiplexing (SM) technique and linear minimum mean square error (LMMSE) detection method with the assistance of the hamming code as well as the interleaving techniques for a better enhanced performance of an audio transmission. Moreover, the comparison was done between the two systems for different antenna configurations and with the presence of two types of modulation: binary phase shift key and quateradure phase shift key. These systems are employed by Matlab simulation to show significant results in terms of enhancing the Rayleigh fading channel capacity, bit error rate (BER) and security as well as in recovering the transmitting audio signals. Each system has advantages than the others in one performance term respect to the other terms. The simulation results have provided to prove and discuss our analysis.
\end{abstract}

This is an open access article under the CC BY-SA license.

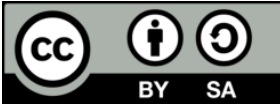

Corresponding Author:

Marwa Al-Sultani

Department of Communications Engineering

University of Diyala, Diyala, Iraq

Email: eng85marwa@gmail.com

\section{INTRODUCTION}

With the developing of the technology and growing the number of the users as well as increase their demands for high speed of internet services and the wireless multimedia providing such as audio, image ...etc lead to rising the efforts to mitigate these limitations through developing the wireless communication system [1-3]. In addition, there are different challenges facing the wireless communication system especially at the receiver ends such as channel fading, interference and other channel noise phenomena $[4,5]$. Thus, the utilizing of multiple antennas at the transmitter ends and receiver ends through applying the diversity techniques in order to enhance the system data rate is a significant solution to mitigate the impairments of the wireless channel which it is having the main role to reduce the capacity of wireless communications system $[6,7]$. The spatial multiplexing could transmit several independent data streams through multipath channels without required increasing bandwidth in terms of achieving a high data rate. LMMSE method could estimate the transmitted signal via combining the linear weight of received version of the signals [8, 9]. However, LMMSE method is complex [10]. It could provide an efficient method to detect the signal at the receiver [11, 12]. Therefore, according to $[13,14]$ this method introduces an important tradeoff between the complexity and the performance.

This paper focuses on improving the channel capacity of transmitting audio signal utilizing SM at the end of the sender and LMMSE method at the accepter. Furthermore, two constellation scenarios, as well 
as various antennas configurations, are implemented in terms of the comparison of the results, one for use the BPSK and the other for QPSK. The observed results illustrate enhancements in terms of Rayleigh fading channel capacity and bit error rate (BER).

There are many papers were covered numerous detection methods to enhance the performing of the multimedia transmission. Wherein [15], this paper uses the least square (LS) channel estimation for a better data rate. Moreover, the researcher in [1], utilizes recursive least squares (RLS) providing better performance than LS. While in [4], they suggest an advanced minimum mean square error successive interference cancellation (MMSE-SIC) detection technique which outperforms other detection such as the conventional zero-forcing, MMSE and the maximum likelihood detections. Employing the Compressive Sensing based Least Square channel estimation technique (LS-CS) at the receiver will improve the quality of the recovered audio signal. In addition, the [16] introduces an equalizer that could perform like a conventional LMMSE detector but with less complexity. This paper is proved improvement in the BER values via choosing modulation methods on every speed level. In addition, analyzing the performance of the channel utilized to transmit audio signals is studied. [17]. This paper is proposed an efficient algorithm called parallel processing decoding technique to achieve spatial diversity. In addition, this proposed algorithm is implemented without using STC [18]. This paper is organized into four sections, Section 2 describes the theoretical analysis of encoder and decoder methods. Section 3 is discussed and analyzed the simulation results. Finally, Section 4 concludes this paper.

\section{RESEARCH METHOD}

This section is employing to describe the system model for both transmitter and receiver as depicted in Figure 1. The input data is providing from recording audio as a signal source then it is converted to corresponding bits by analog to digital converter. The code words are generated after applying a sequence of bits to the channel code by utilizing linear block code (LBC) particularly hamming code $(7,4)[19]$ which have the ability to correct one error and detect two errors [20, 21]. Hence, the coded information bits are interleaved by two dimensions interleaving that the interleaving index have the same length with the coded messages which is seven binary digits to decrease the complexity after that they are modulated digitally by BPSK for first scenario and QPSK for the second scenario. The modulating symbols are fed into a spatial multiplexer (SM) that have $N_{t}$ antennas to be transmitted over flat fading channel under Rayleigh distribution environments. On the other hand, in the received section, the linear minimum mean square error method (LMMSE), that have $N_{r}$ antennas will detect the transmitted signals. The signals are demodulated, deinterleaved and channel decoded to recover the audio signals.

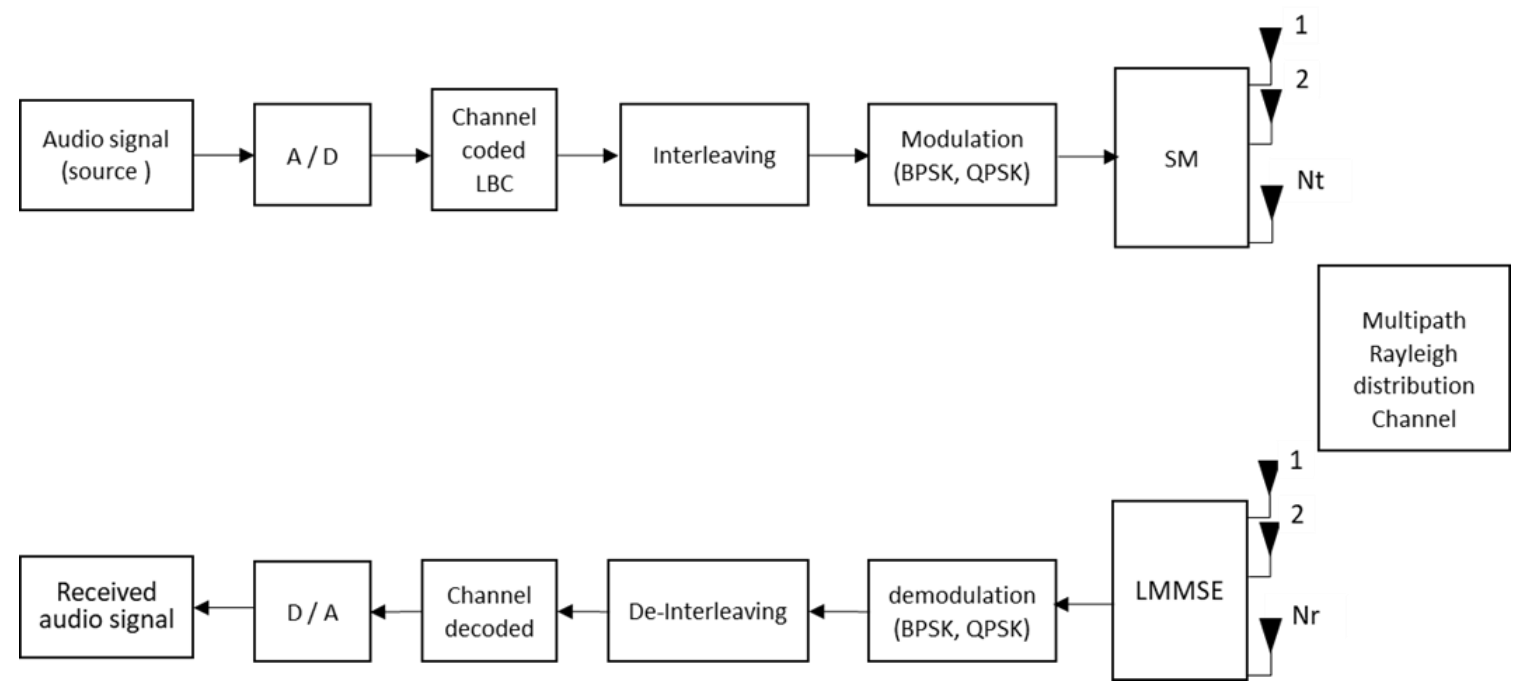

Figure 1. The system model

The signals arrived from the transmitter to the receiver suffered from scattering because of the multipath environment. The spatial multiplexing (SM) is used at the transmitter where the input signal is obtained from an audio signal then the information bits $(k)$ is treated by LBC to generate $(n)$ bits of a 
codeword and then interleaved [22-24]. The modulated signal is splitting as a layer into $N_{t}$ data stream having different rates where higher-order if it has a higher data rate, as a result, each one of the layers might own the same bandwidth [25].

$$
n_{\text {stream }}=\min \left\{N_{t}, N_{r}\right\}
$$

Where $n_{\text {stream }}$ represents the independent data steam number transmitting over SM. $N_{t}, N_{r}$ is the number of transmitted and received antennas, respectively. The efficiency of spatial multiplexing (SM) could be obtained as

$$
\gamma_{\max }=N_{t} R_{t} \log _{2}(m) b p s / H z
$$

Where $\gamma_{\max }$ denotes to the maximum spectral efficiency of the spatial multiplexing and $R_{t}$ and $\mathrm{m}$ represent the rate of the $\mathrm{LBC}$ and modulation order, respectively.

Considering a system have respectively $N_{t}, N_{r}$ antennas at both ends used horizontal ball Laboratory layered space-time (H-BLAST) as SM technique so the symbol that sent at any point in time $(q)$ for each transmitter antenna $(j)$ could name as $x_{j}(q)$. The received signal $r_{i}(q)$ is received at the receiver $(i)$ with the amplitude noise $z_{i}(q)$ during the same period time of transmitting symbol with $p$ that represents the average of SNR. In addition, the $H$ represents the $N_{r} \times N_{t}$ channel matrix.

At the receiver terminals, the linear minimum mean square error method (LMMSE) is applied. This method could estimate the transmitted signal by combining the linear weight of the received version of the signals. In this method, the $w_{o}$ is a matrix which it is responsible to reduce the mean square error (MSE) between the real signal $(x)$ and estimated one $(\hat{x})$ so the equation could illustrate as

$$
w_{o}=\frac{\sqrt{p}}{N_{t}} H^{H}\left(\frac{p}{N_{t}} H H^{H}+I_{N_{t}}\right)^{-1}
$$

Representing $H^{H}$ the Hermitian operation of a channel matrix, and $I_{N_{t}}$ is the identity matrix. Therefore; the received vector is

$$
\tilde{Y} \triangleq w_{o} Y
$$

Therefore; the estimated received signal at the time $q$ of the $j t h$ row is

$$
\begin{aligned}
& \tilde{y}_{j}(q)=\sqrt{p} \sum_{m=1}^{N_{r}} w_{j, m} \sum_{n=1}^{N_{t}} h_{m, n} x_{n}(q)+\tilde{Z}_{j}(q) \\
& \tilde{y}_{j}(q)=\sqrt{p}\left(\sum_{m=1}^{N_{r}} w_{j, m} h_{m, j}\right) x_{j}(q)+\sqrt{p} \sum_{m=1}^{N_{r}} w_{j, m} \sum_{n \neq j}^{N_{t}} h_{m, n} x_{n}(q)+\tilde{Z}_{j}(q)
\end{aligned}
$$

By normalized (6) as (7), the estimated signal is (8)

$$
\begin{aligned}
& \hat{y}_{j}(q) \triangleq \frac{\tilde{y}_{j}(q)}{\sqrt{p} \sum_{m=1}^{N_{r}} w_{j, m} h_{m, j}} \\
& \hat{y}_{j}(q)=x_{j}(q)+\frac{\sum_{m=1}^{N_{r}} w_{j, m} \sum_{n \neq j}^{N_{t}} h_{m, n} x_{n}(q)}{\sum_{m=1}^{N_{r}} w_{j, m} h_{m, j}}+\frac{\tilde{z}_{j}(q)}{\sqrt{p} \sum_{m=1}^{N_{r} w_{j, m} h_{m, j}}}
\end{aligned}
$$

From (8), this estimated received signal depends on the transmitted signal which is sent through $j$ th transmit antenna, but this extracted signal is associated with interference and noise terms which are stated in the second and third terms in (8), respectively. Then, the maximum likelihood estimate can be applied on (8) to estimate the transmitted signal.

$$
\widehat{x}_{j}(q)=\arg \min \left|\hat{r}_{j}(q)-x_{j}(q)\right|^{2} j=1, \ldots, N_{t} q=1, \ldots, p
$$

This method performs the better of the linear SM detection and decoding methods while the other method suffers from noise amplification. 


\section{RESULTS AND DISCUSSION}

This section presents, analyzes, and discusses the simulation result that is implemented utilizing MATLAB simulation. Computing has occurred via employed two scenarios. The first scenario is implemented by using BPSK and four types of antenna configuration while the second one is used QPSK instead of the BPSK. The simulation parameters are tabulated in a Table 1.

Table 1. Simulation parameters

\begin{tabular}{ll}
\hline Description of Parameter & Value \\
\hline Data transmitted & Audio signal \\
Configuration of antenna & $(1 \times 1),(2 \times 2),(4 \times 4)$ and $(8 \times 8)$ \\
SM technique & H-BLAST \\
Channel Coding & LBC (Hamming code) \\
Interleaving & Two-dimensional interleaving \\
Interleaving index & 7 \\
Modulation and demodulation type & BPSK and QPSK \\
Signal detection method & LMMSE \\
Channel type & Rayleigh fading Multipath \\
Noise type & Gaussian noise or AWGN \\
SNR $(\mathrm{dB})$ & -3 to 10 \\
\hline
\end{tabular}

This research focuses on increasing the channel capacity using spatial multiplexing (SM) technique at the transmitter that enables to send of independent modulated symbols at a single time $\mathrm{q}$. The number of these modulated symbols depends on the minimum number of the transmitted and received antennas as stated in (1) [25]. Figure 2 shows a Rayleigh fading channel capacity for four different types of configurations using BPSK.

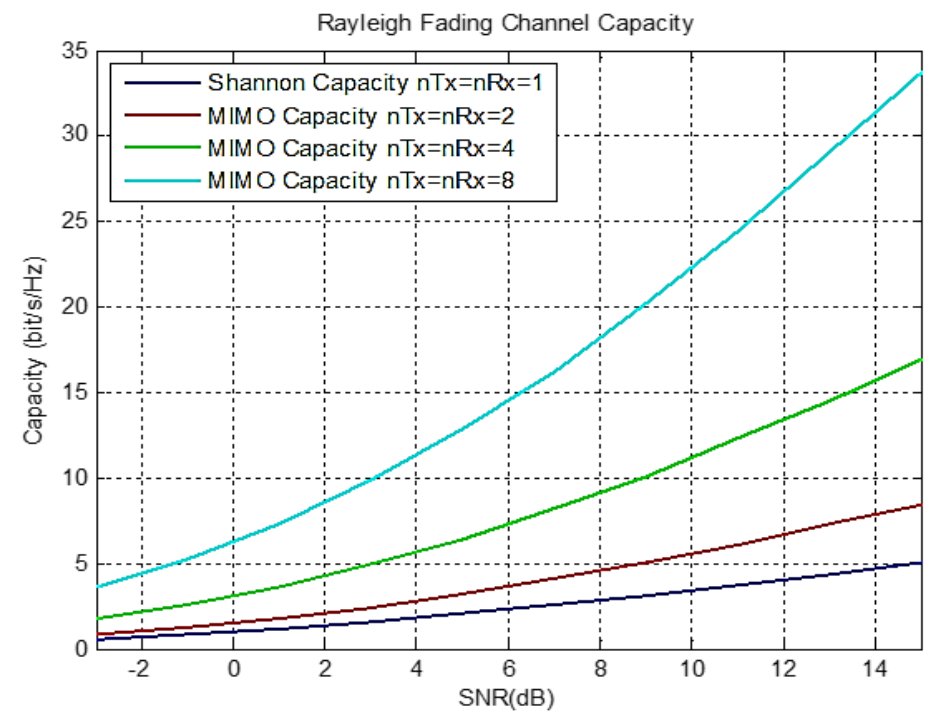

Figure 2. Rayleigh fading channel capacity at BPSK

The capacity of using eight antennas at both ends of the transmitter and the receiver is the best of the other configurations in the same type of constellation which is BPSK modulation [6]. However; the second scenario deals with the other type which is QPSK modulation. It is known that the data rate of QPSK is twice of BPSK. Furthermore, the capacity performance of the $(4 \times 4)$ configuration employing QPSK is the same as the $(8 \times 8)$ employing BPSK.

On the other side, the second parameter that is improved in this research is the bit error rate (BER). In both scenarios, BER of audio transmission is reduced utilizing the LMMSE method. Figure 3 shows the performance of both scenarios where it is recognizable that the system works better in BPSK than QPSK. 


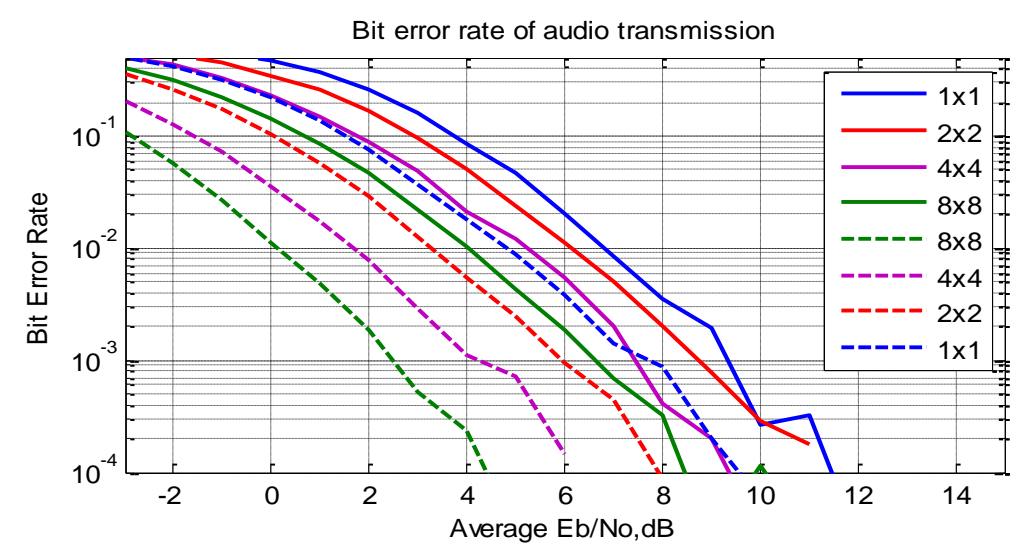

Figure 3. System performance comparison at both scenarios: BPSK and QPSK

The $(8 \times 8)$ configuration is better performing in the same constellation. It is obviously an increase in the antenna numbers leading to decrease BER due to employing the LMMSE method as shown in Figure 3 which straight line represents QPSK and dash line represents BPSK. This performance even in $(2 \times 2)$ configuration for both types of PSK modulations is a better than the simulated results in [4] because of employing hybrid techniques of LMMSE detection, LBC, and interleaving technique which is randomized the sequential bits of the audio signal in which increasing the capability of LBC to detect and correct more bit errors [22]. In [4], the authors enhanced LMMSE-SIC technique while it is more complicated than LMMSE technique. However, the BER term of the proposed system in both scenarios is clearly less than the advanced MMSE-SIC technique.

Furthermore, there is more enhancement applying BPSK than QPSK in this research due to implementing grouped of LBC and two dimensional bit interleaved. There is a trade-off between enhanced terms. For instance, in Figure 3, the dashed blue curve which is $(1 \times 1)$ BPSK performs the same of the purple curve which is $(4 \times 4)$ QPSK in terms of BER while, in Figure 2, the channel capacity of the second one is efficiently the more. However; the dashed green curve which is $(8 \times 8)$ BPSK performs dramatically better than the purple curve which is $(4 \times 4)$ QPSK while both of these two systems achieved the same channel capacity. Figure 4 presents the original transmitted audio signal. It will be utilized to compare with the other results from Figures 5 and 6 which denote the received audio signal for various SNR and both scenarios.

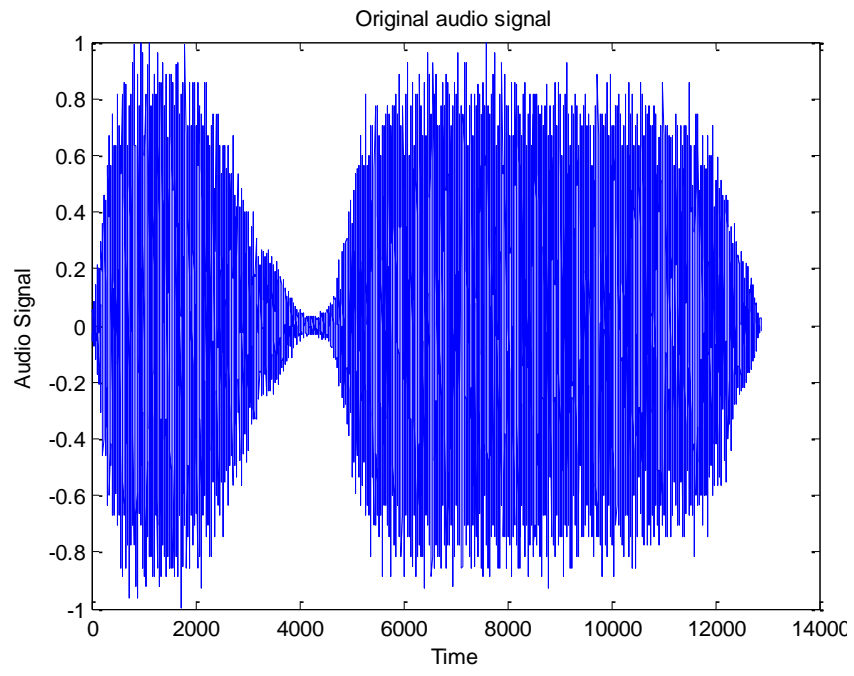

Figure 4. Original audio signal

Figures 5 and 6 introduce the received audio signals at SNR value of $5 d B$ implemented in the both two scenarios and apply for different antenna configurations. 


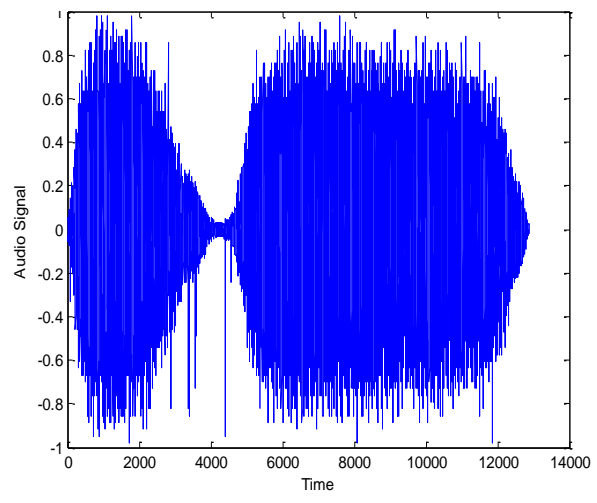

(a) $n T x=n R x=1$

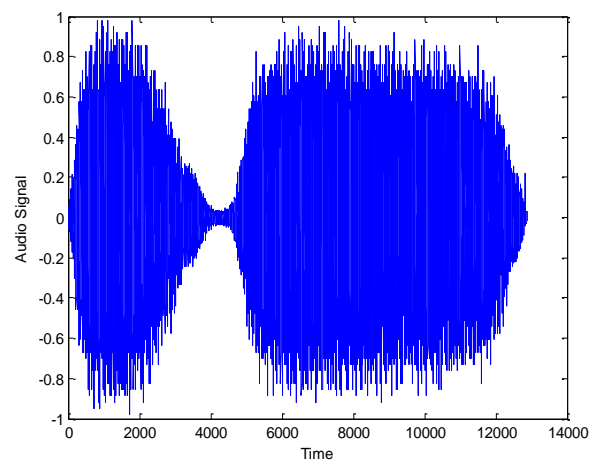

(c) $n T x=n R x=4$

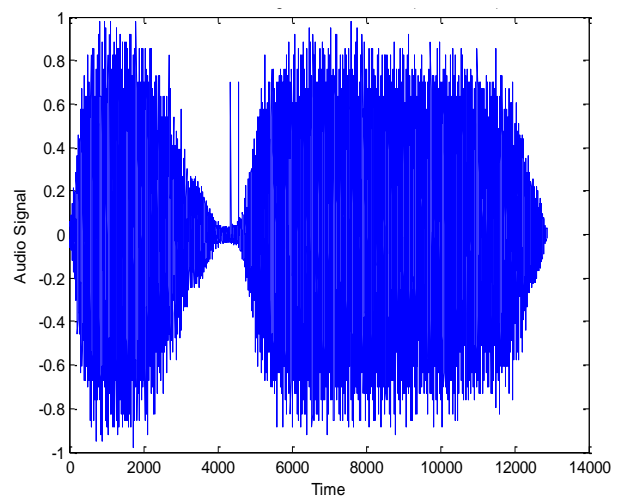

(b) $n T x=n R x=2$

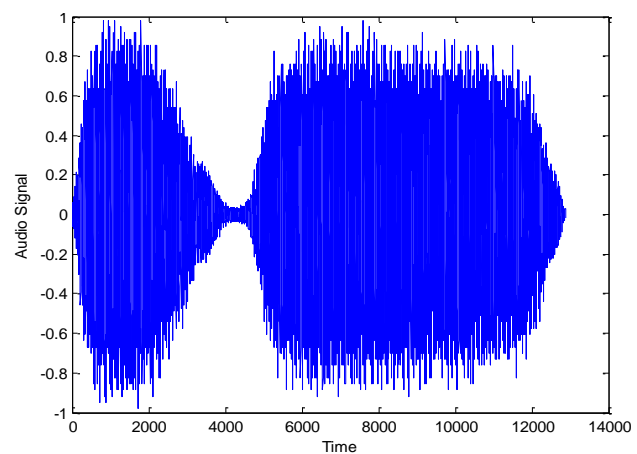

(d) $n T x=n R x=8$

Figure 5. Received audio signals at $\mathrm{SNR}=5 \mathrm{~dB}$ using BPSK

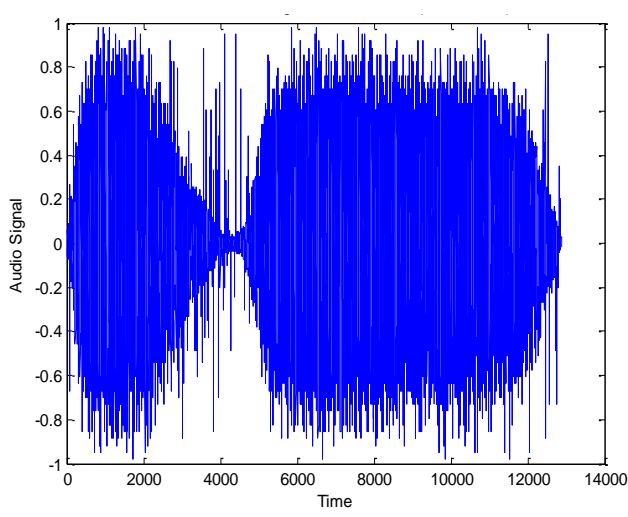

(a) $n T x=n R x=1$

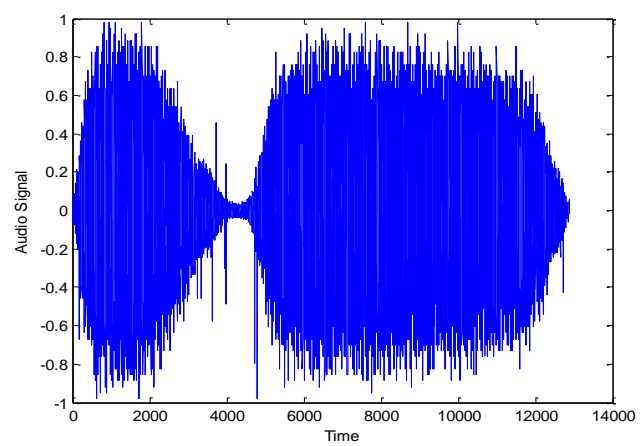

(c) $n T x=n R x=4$

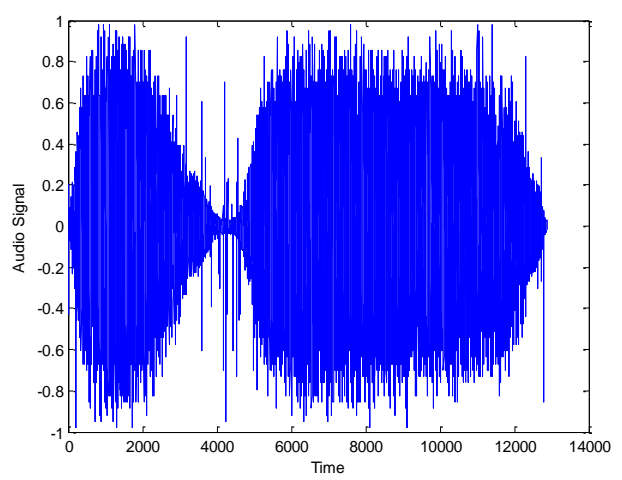

(b) $n T x=n R x=2$

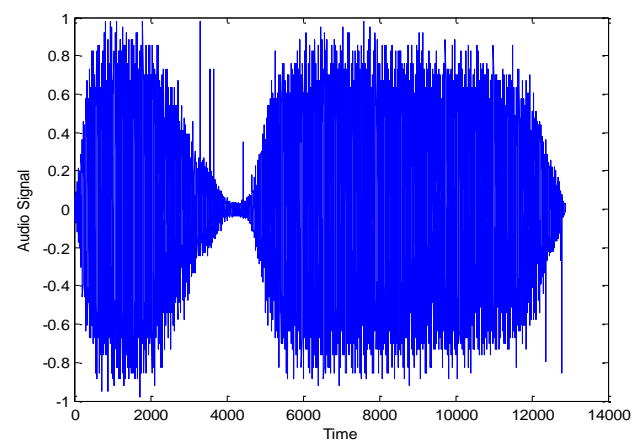

(d) $n T x=n R x=8$

Figure 6. Received audio signals at $\mathrm{SNR}=5 \mathrm{~dB}$ using QPSK 
In the both figures, the extracted audio signals are almost identical to the original audio signal with increasing the number of antennas at both ends. It is notable, it is great estimated audio signals at a typical value of SNR $10 \mathrm{~dB}$ for all antenna configurations in both two scenarios.

\section{CONCLUSION}

This research establishes a model system to enhance the performance of the audio transmission signal. Hence, the proposed system is based on the LMMSE detection method to mitigate the limitation result from the multipath environments of the channel. However, a comparison in results is accrued through the implementation of two kinds of constellations (BPSK and QPSK) applying for four types of antenna configurations. The simulation results present a better enhancement in the audio transmission than other results in comparison papers specifically with the presence of spatial multiplexing and the LMMSE decoding method with aiding of LBC as a coding channel and interleaving technique. Furthermore, the Rayleigh fading channel capacity and bit error rate (BER) had been improved leading to better performance of the system. There is a trade-off relation between these two terms of the performance as stated in more detail in the results and discussion section. Each system of these two scenarios has advantages than the others in one performance term respect to the other terms. Where the $(8 \times 8)$ QPSK system performs the greatest channel capacity with lower BER enhancement than the others. On the other hand, the $(8 \times 8)$ BPSK system performs the best in terms of BER enhancement, but its channel capacity is the same as $(4 \times 4)$ QPSK system. Related future work can be investigated more techniques or made hybrid techniques to get an optimized system that manipulates the capacity, BER, security, complexity, cost, ... etc.

\section{REFERENCES}

[1] Y. Liang, et al, "RLS channel estimation and data detection in space-time coded MIMO-OFDM systems," in 2006 First International Conference on Communications and Networking in China, pp. 1-5, 2006.

[2] B. Das, M. P. Sarma, and K. K. Sarma, "Different aspects of interleaving techniques in wireless communication," in Intelligent Applications for Heterogeneous System Modeling and Design: IGI Global, pp. 335-374, 2015.

[3] R. Bhandari and S. Jadhav, "Spectral efficient blind channel estimation technique for mimo-ofdm communications," International Journal of Advances in Applied Sciences (IJAAS), vol. 7, no. 3, pp. 286-297, 2018.

[4] S. Sarkar, "An advanced detection technique in MIMO-PSK wireless communication systems using MMSE-SIC detection over a Rayleigh fading channel," Csi Transactions on Ict, vol. 5, no. 1, pp. 9-15, 2017.

[5] A. M. Elshirkasi, et al, "Performance evaluation of 2-port MIMO LTE-U terminal antenna with user's hand effect," Bulletin of Electrical Engineering and Informatics, vol. 8, no. 1, pp. 158-165, 2019.

[6] M. Al-Sultani, W. Q. Mohamed, and M. Huiyi, "Study on Various Antenna Configurations," in 2018 2nd International Symposium on Multidisciplinary Studies and Innovative Technologies (ISMSIT), pp. 1-5, 2018.

[7] X. Liu, et al, "A low complexity high performance weighted neumann series-based Massive MIMO detection," in 2019 28th Wireless and Optical Communications Conference (WOCC), pp. 1-5, 2019.

[8] X. Yuan, C. Xu, L. Ping, and X. Lin, "Precoder design for multiuser MIMO ISI channels based on iterative LMMSE detection," IEEE Journal of Selected Topics in Signal Processing, vol. 3, no. 6, pp. 1118-1128, 2009.

[9] J. P. Patra and P. Singh, "A novel LMMSE-EM channel estimator for high mobility STBC-OFDM system," Journal of Circuits, Systems and Computers, vol. 28, no. 13, p. 1950223, 2019.

[10] L. Fang and D. Huang, "Neumann series expansion based LMMSE channel estimation for OFDM systems," IEEE Communications Letters, vol. 20, no. 4, pp. 748-751, 2016.

[11] L. Ge, Y. Zhang, G. Chen, and J. Tong, "Compression-based lmmse channel estimation with adaptive sparsity for massive MIMO in 5G systems," IEEE Systems Journal, vol. 13, no. 4, pp. 3847-3857, 2019.

[12] P. Sen and A. Ö. Y1lmaz, "A low-complexity graph-based LMMSE receiver for MIMO ISI channels with \$M \$QAM modulation," IEEE Transactions on Wireless Communications, vol. 16, no. 2, pp. 1185-1195, 2016.

[13] L. Liu, C. Yuen, Y. L. Guan, and Y. Li, "Capacity-achieving iterative LMMSE detection for MIMO-NOMA systems," in 2016 IEEE International Conference on Communications (ICC), pp. 1-6, 2016.

[14] M. Tuchler, A. C. Singer, and R. Koetter, "Minimum mean squared error equalization using a priori information," IEEE Transactions on Signal processing, vol. 50, no. 3, pp. 673-683, 2002.

[15] S. J. Lee, "Effect of least square channel estimation errors on achievable rate in MIMO fading channels," IEEE Communications Letters, vol. 11, no. 11, pp. 862-864, 2007.

[16] X. He, Q. Guo, J. Tong, J. Xi, and Y. Yu, "Low-complexity approximate iterative LMMSE detection for large-scale MIMO systems," Digital Signal Processing, vol. 60, pp. 134-139, 2017.

[17] J. Hendry, et al, "Audio signal transmission over vehicular channel with moving scatterer," in 2019 International Conference of Artificial Intelligence and Information Technology (ICAIIT), pp. 490-495, 2019.

[18] W. Q. Mohamed and K. Hamidian, "Reliability enhancement without employing STCs of a $3 \times 3$ MIMO communication system using a parallel decoding technique," in 2018 International Conference on Engineering Technology and their Applications (IICETA), pp. 91-95, 2018.

[19] C.-Y. Lin, Y.-C. Huang, S.-L. Shieh, and P.-N. Chen, "Transformation of binary linear block codes to polar codes with dynamic frozen," IEEE Open Journal of the Communications Society, vol. 1, pp. 333-341, 2020. 
[20] R. Avudaiammal, Information coding techniques, Tata McGraw Hill Education, 2010.

[21] W. Lei, G. et al, "The Method to recognize linear block code based on the distribution of code weight," in 2018 10th International Conference on Communication Software and Networks (ICCSN), pp. 16-20, 2018.

[22] Y. Cui and X. Zhang, "Research and implemention of interleaving grouping Hamming code algorithm," in 2013 IEEE International Conference on Signal Processing, Communication and Computing (ICSPCC 2013), pp. 1-4, 2013.

[23] A. F. Fadlilah and E. C. Djamal, "Speaker and speech recognition using hierarchy support vector machine and backpropagation," in 2019 6th International Conference on Electrical Engineering, Computer Science and Informatics (EECSI), pp. 404-409, 2019.

[24] V. V. Bolisetty, U. Yedukondalu, and I. Santiprabha, "Speech enhancement using modified wiener filter based MMSE and speech presence probability estimation," International Journal of Informatics and Communication Technology (IJ-ICT), vol. 9, no. 2, pp. 63-72, 2020.

[25] J. R. Hampton, Introduction to MIMO communications, Cambridge university press, 2013.

\section{BIOGRAPHIES OF AUTHOR}

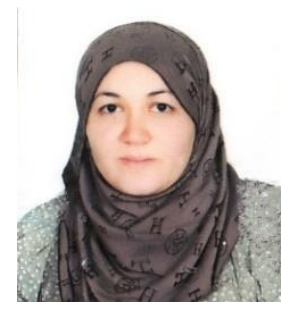

Marwa Mohammed Jawad teaching in the department of communication engineering $\backslash$ college of engineering\ University of Diyala. She takes on the bachelor's degree in the field of communication engineering from university of Diyala\Iraq, (2006), and she takes master's degree (2017) at the Telecommunication engineering from University of New South Wales (UNSW) \Australia. She works from year (2007-2014) as an engineer at department of communication engineering luniversity of Diyala \Iraq and works as assistant lecture from year (2017 up to date) at department of communication engineering \ college of engineering \ University of Diyala. Research Interests: Wireless communication technology, mobile and satellite communication system, coding and information theory

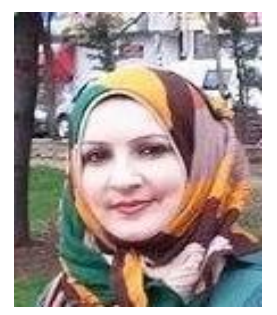

Lecturer Haraa Raheem Hatem teaching in the department of communication engineering I college of engineering \University of Diyala. She takes on the bachelor's degree in the field of electronic engineering from university of Diyala (2002), and she takes master's degree (2014) at the field of Electronics and Communications engineering from department of electric engineering luniversity of Al-Mustanseriya \Baghdad. She worked as engineer (2003-2011) at department of communication engineering \ college of engineering $\backslash$ University of Diyala. She works as teaching (Assistant lecturer) from year (2014 up to date) at department of communication engineering \ college of engineering \University of Diyala. Research Interests: Image Processing, Encryption, Control and Digital Signal Processing and OFDM

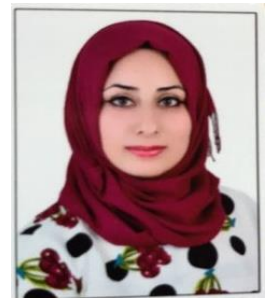

Wurod Qasim Mohamed is a lecturer of Communications Engineering at Department of Electronic Engineering / College of Engineering / University of Diyala. She received her B.S of Electronics Engineering from College of Engineering / University of Diyala, Iraq in 2011, and she received her M.S. from California State University Fullerton (CSUF), CA, and USA in Fall 2016 semester after getting scholarship from High Committee for Education Development (HCED) in Iraq. During her study master degree, she taught various electronics and communications courses at CSUF for three semesters. Research Interests: Wireless communications systems, OFDM techniques, MIMO techniques, MIMO - OFDM communications systems and Signal processing. 